\title{
High-resolution spectroscopy of two LBV cycles of HR Car
}

\author{
Thomas Szeifert ${ }^{1}$, Andreas Kaufer ${ }^{1}$, Paul A. Crowther ${ }^{2}$, Otmar Stahl ${ }^{3}$, \\ and Chris Sterken ${ }^{4}$ \\ ${ }^{1}$ European Southern Observatory, \\ Alonso de Cordova 3107, Santiago 19, Chile \\ ${ }^{2}$ Department of Physics and Astronomy, University College London, \\ Gower Street, London WC1E 6BT, UK \\ ${ }^{3}$ Landessternwarte Königstuhl, D-69117 Heidelberg, BRD \\ ${ }^{4}$ Astrofysisch Instituut, Vrije Universiteit Brussel, \\ Pleinlaan 2, B-1050 Brussel, België
}

\begin{abstract}
Luminous Blue Variables (LBVs or SDor Variables) are showing characteristic variability of very long timescales of a decade and more. During their expansion and contraction phases, they move across a wide part of the H-R diagram with dramatic changes of their stellar wind characteristics, radius and temperature. We present results of our long-term spectroscopic monitoring campaigns for the galactic star HR Car with optical spectra taken over 13 years and two minimum and maximum phases observed during this time.
\end{abstract}

HR Car has shown early B-type hypergiant spectra characterized by Si II and Si III absorption lines, as well as strong N II during two epochs: in 1989 and between 1993 and 1996. Until early 1993, the Balmer lines with P-Cygni profiles became stronger, indicating increasing densities in the wind, due to increasing mass-loss rates. Until the end of 1991, the star was cooling down to a lateB or early-A spectral type. All strong Fe I and and CrII lines are during this phase in emission or are showing pronounced P-Cygni profiles. Since 1997, the star is cooling down again and has reached the for S Dor variables characteristic photometric maximum phase in 1999, which extends over a longer time span then the 1993 event. With the help of the visual estimates of the RASNZ observers, the phases can be nicely associated with photometric maxima in 1991 and a still ongoing maximum phase since late 1999. Indicated by the appearance of the Ti II lines, the lower-most temperatures are only reached during the recent LBV maximum phase, while the expansion got suddenly interupted during the event in 1993.

During the hot minimum phases the absorption lines of Si III or N II are broad and can be reproduced with rotation profiles of about $70 \mathrm{~km} \mathrm{~s}^{-1}$. At this time, the Balmer lines show discrete multiple components with wind velocities up to $200 \mathrm{~km} \mathrm{~s}^{-1}$ and the peak flux in line-emission red-shifted with respect to what was observed during the cold maximum phases. Possibly rotation might cause axial-symmetric components in the stellar wind of HR Car and, therefore, this star is a very special but also very complex member of the small group of S Dor variables. The Mg II $4481 \AA$ line, observable in A-type as well as in early B-type 
spectra, is clearly variable, not only in line strength, but also in line width. Even though the line is strongly asymmetric, it is still an interesting tendency to see this line narrow during the phase of maximum expansion in 1999, well consistent with a spin-down of the expanding LBV atmosphere. Pulsation-like motion (micro-variability) can maybe contribute, but not explain, the extreme absorption line widths. The stellar wind might also contribute to the line broadening, but primarely on the blue edge of the profile.

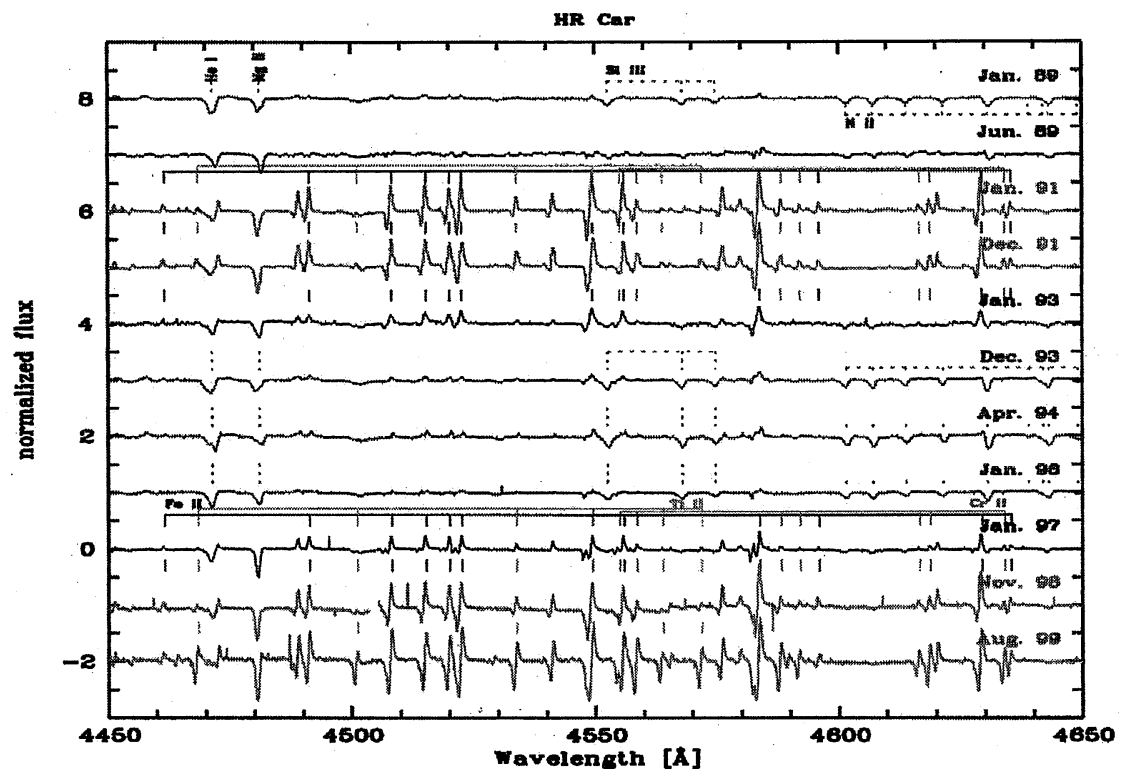

Figure 1. Long-term time-series of HR Car. He I, Mg II, Si III and N II absorption lines characterize the spectral appearance in June 89 and between 1993 and 1996. During the cold phases Ti II, Cr II and Fe II lines with P-Cygni profiles are observed. 